\title{
Distributed glycine in comet 67P/Churyumov-Gerasimenko
}

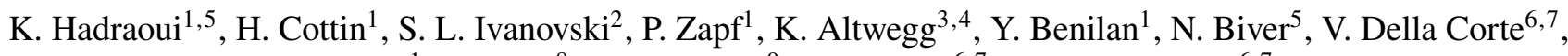 \\ N. Fray ${ }^{1}$, J. Lasue ${ }^{8}$, S. Merouane ${ }^{9}$, A. Rotundi ${ }^{6,7}$, and V. Zakharov ${ }^{6,7}$ \\ ${ }^{1}$ Laboratoire Interuniversitaire des Systèmes Atmosphériques (LISA), UMR CNRS 7583, Université Paris-Est-Créteil, Université de \\ Paris, Institut Pierre Simon Laplace (IPSL), Créteil, France \\ e-mail: katia.hadraoui@lisa.u-pec.fr \\ 2 INAF - Osservatorio Astronomico di Trieste, Via Tiepolo 11, 34143 Trieste, Italy \\ ${ }^{3}$ Physikalisches Institut, University of Bern, Sidlerstrasse 5, 3012 Bern, Switzerland \\ ${ }^{4}$ Center for Space and Habitability, University of Bern, Sidlerstrasse 5, 3012 Bern, Switzerland \\ ${ }^{5}$ LESIA, Observatoire de Paris, Université PSL, CNRS, Sorbonne Université, Université Paris Diderot, Sorbonne Paris Cité, \\ 5 place Jules Janssen, 92195 Meudon, France \\ ${ }^{6}$ INAF - Istituto di Astrofisica e Planetologia Spaziali, Ricerca Tor Vergata, Via Fosso del Cavaliere 100, 00133 Rome, Italy \\ ${ }^{7}$ Università degli Studi di Napoli Parthenope, Dipartimento di Scienze e Tecnologie, CDN IC4, 80143 Naples, Italy \\ ${ }^{8}$ IRAP, Université de Toulouse, CNRS, UPS, Toulouse, France \\ ${ }^{9}$ Max-Planck-Institut für Sonnensystemforschung, Justus-von-Liebig-Weg 3, 37077 Göttingen, Germany
}

Received 4 January 2019 / Accepted 18 May 2019

\begin{abstract}
Most of the gaseous molecules that are detected in cometary atmospheres are produced through sublimation of nucleus ices. Distributed sources may also occur, that is, production within the coma, from the solid component of dust particles that are ejected from the nucleus. Glycine, the simplest amino acid, was observed episodically in the atmosphere of comet 67P/Churyumov-Gerasimenko (67P) by the ROSINA mass spectrometer on board the Rosetta probe. A series of measurements on 28 March 2015 revealed a distributed density profile at between 14 and $26 \mathrm{~km}$ away from the nucleus. We here present and discuss three study cases: (i) glycine emitted directly and only from the nucleus, (ii) glycine emitted from the sublimation of solid-state glycine on the dust particles that are ejected from the nucleus, and (iii) glycine molecules embedded in water ice that are emitted from the sublimation of this ice from the dust particles that are ejected from the nucleus. A numerical model was developed to calculate the abundance of glycine in the atmosphere of comet 67P as a function of the distance from the nucleus, and to derive its initial abundance in the lifted dust particles. We show that a good fit to the observations corresponds to a distributed source of glycine that is embedded in sublimating water ice from dust particles that are ejected from the nucleus (iii). The few hundred ppb of glycine embedded in water ice on dust particles (nominally 170 ppb by mass) agree well with the observed distribution.
\end{abstract}

Key words. comets: individual: 67P/Churyumov-Gerasimenko - astrochemistry

\section{Introduction}

Glycine $\left(\mathrm{NH}_{2}-\mathrm{CH}_{2}-\mathrm{CO}_{2} \mathrm{H}\right)$ is the simplest amino acid and is a building block of proteins. Although realistic prebiotic chemical pathways from amino acids to proteins are still being investigated, glycine is often considered as being involved in the prebiotic chemistry that led to the origin of life on Earth. The source of amino acids to the primitive Earth is still an open question. Whether they could be produced in the Earth's early atmosphere or in hydrothermal vents depends on the composition of the atmosphere and oxidation state of the upper mantle (Cottin et al. 2017). However, it has been demonstrated that amino acids have been imported to some extent to Earth from extraterrestrial material. In 1970, glycine was first unambiguously detected in the Murchison meteorite (Kvenvolden et al. 1970) with an abundance of about 3000 ppb (Martins \& Sephton 2009), and since then, it was discovered in numerous carbonaceous chondrites (Martins \& Sephton 2009). Its concentration is usually of a few tens to a few thousand ppb by mass most in CI and CM chondrites (Martins \& Sephton 2009; Burton et al. 2015). Glycine has also been detected in comets. First, in dust particles collected from comet 81P/Wild 2 and returned to Earth during the Stardust mission in 2004 (Elsila et al. 2009). Then, in situ detection was reported in the atmosphere of comet 67P/Churyumov-Gerasimenko (hereafter 67P) during the Rosetta mission (Altwegg et al. 2016). On 28 March 2015, a glycine density profile was measured at between 14 and $26 \mathrm{~km}$ away from the nucleus of 67P with the Rosetta Orbiter Spectrometer for Ion and Neutral Analysis (ROSINA) on board the Rosetta probe (Altwegg et al. 2016). It can be deduced from these measurements that its distribution cannot be interpreted by direct sublimation from the nucleus (Fig. 1a) (hereafter referred to as case 1), but rather from a distributed source (Altwegg et al. 2016). This means that there is an additional source of glycine that is produced from the dust particles that are ejected from the nucleus, which releases glycine in the gaseous phase as they move outward in the coma. Other distributed sources have previously been observed in comets for molecules such as $\mathrm{H}_{2} \mathrm{CO}$ and for $\mathrm{HNC}$ or $\mathrm{CN}$, and processes such as the thermal or photodegradation of organic material in the solid state on dust particles have been evoked as an interpretation (see Cottin \& Fray 2008 for a review or Cordiner et al. 2014 
Table 1. Glycine and water parameters.

\begin{tabular}{cc}
\hline \hline Glycine & Water \\
\hline$L=137100 \mathrm{~J} \mathrm{~mol}^{-1}$ (De Kruif et al. 1979) & $L=52000 \mathrm{~J} \mathrm{~mol}^{-1}$ (Wagner et al. 2011) \\
$P_{\text {ref }}=0.4 \mathrm{~Pa}$ (De Kruif et al. 1979) & $P_{\text {ref }}=611.657 \mathrm{~Pa}$ (Wagner et al. 2011) \\
$T_{\text {ref }}=419.92 \mathrm{~K}$ (De Kruif et al. 1979) & $T_{\text {ref }}=273 \mathrm{~K}$ (Wagner et al. 2011) \\
$\rho_{\text {Gly }}=1.61 \times 10^{6} \mathrm{~g} \mathrm{~m}^{-3}$ & $\rho_{\mathrm{Gly}}=1.61 \times 10^{6} \mathrm{~g} \mathrm{~m}^{-3}$ \\
$J_{\mathrm{Gly}}=10^{-2} \mathrm{~s}^{-1}$ (Saiagh et al. 2015) & $J_{\mathrm{Gly}}=10^{-2} \mathrm{~s}^{-1}$ (Saiagh et al. 2015) \\
$Q_{\text {gas }}=5 \times 10^{26} \mathrm{molec} \mathrm{s}^{-1}$ (Biver et al. 2019) & $Q_{\mathrm{gas}}=5 \times 10^{26} \mathrm{molec} \mathrm{s}^{-1}$ (Biver et al. 2019) \\
$V_{\mathrm{g}}=750 \mathrm{~m} \mathrm{~s}^{-1}$ (Biver et al. 2019) & $V_{\mathrm{g}}=750 \mathrm{~m} \mathrm{~s}^{-1}$ (Biver et al. 2019) \\
\hline
\end{tabular}

Notes. The gas velocity and the total gas abundance correspond to March 2015.

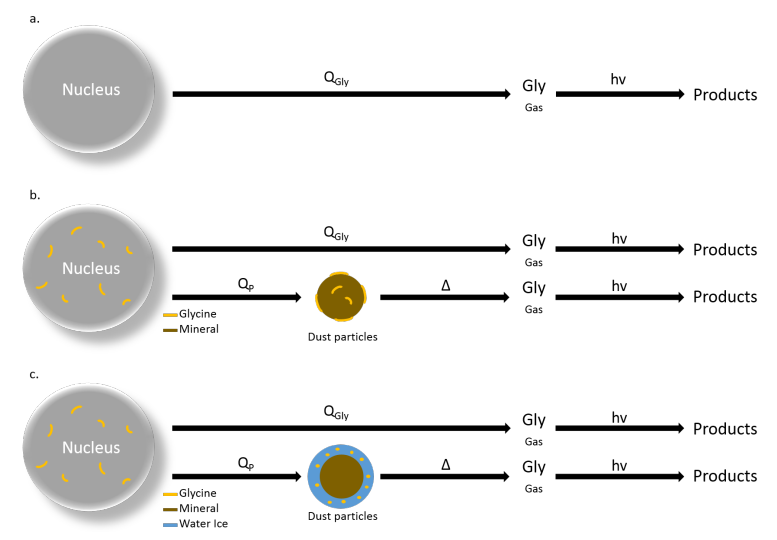

Fig. 1. Three cases of glycine production discussed in this work. Panel $a$ : glycine emitted directly and only from the nucleus. Panel $b$ : glycine emitted from the sublimation of solid-state glycine in the dust particles that are ejected from the nucleus. Panel $c$ : glycine embedded in water ice that is emitted from the sublimation of this ice from the dust particles that are ejected from the nucleus.

for more recent observations of $\mathrm{H}_{2} \mathrm{CO}$ and $\mathrm{HNC}$ distributed sources). In order to assess the validity of a distributed source of glycine in comet 67P, two cases of a distributed source are considered in this paper. In a first case (case 2), glycine is emitted from the sublimation of solid-state glycine on the dust particles that are ejected from the nucleus (Fig. 1b). In this case study, we consider that the dust particles are porous aggregates (Langevin et al. 2016) with $90 \%$ of porosity (Hornung et al. 2016), in which inclusions of glycine molecules, in the solid state, are randomly dispersed at the surface and inside the dust particles. The porosity is sufficiently high so that when it sublimes, glycine readily diffuses and is released into the cometary atmosphere. The sublimation of glycine is then modeled as if it were pure solid-state glycine inclusions at the surface of the dust particles. In another case (case 3), glycine is embedded in water ice and emitted from the sublimation of this water ice from the dust particles that are ejected from the nucleus (Fig. 1c). In this case study, glycine molecules are trapped in the water ice, which is uniformly distributed within the porous dust particles. Like in case 2, the dust particles are considered to be sufficiently porous to allow water and molecules trapped within the ice to diffuse and be released into the atmosphere. Then the release of glycine is modeled following the kinetics of water ice sublimation, as if it were located at the surface of the dust particles, whereas in case 2 the release of glycine follows the kinetics of glycine sublimation. Thus some parameters in Eqs. (1)-(6) differ from one case to another and are referenced in Table 1.
Experiments reported by Bernstein et al. (2002), Caro et al. (2002), Meinert et al. (2012), and Esmaili et al. (2018) have shown that glycine could be produced in laboratory interstellar and cometary ice analogs (simple ice mixtures such as $\mathrm{H}_{2} \mathrm{O}$, $\mathrm{CO}$, and $\mathrm{CO}_{2}$, photolyzed or irradiated at about $10 \mathrm{~K}$ and then warmed to room temperature). From the theoretical point of view, Woon (2002) has suggested that the synthesis of glycine might be explained by the addition of radicals $\mathrm{NH}_{2} \mathrm{CH}_{2}$ and $\mathrm{HOCO}$ that formed by successive hydrogenation of $\mathrm{HCN}$ and by the addition of $\mathrm{CO}$ to $\mathrm{OH}$, respectively. More recently, several studies have proposed other mechanisms for glycine production in the interstellar medium. For instance, Singh et al. (2013) discussed three different reaction paths in the gaseous phase as well as in water-dominated ices that coat interstellar dust,

$$
\begin{aligned}
& \mathrm{NH}_{2}+\mathrm{CH}_{2} \rightarrow \mathrm{NH}_{2} \mathrm{CH}_{2}+\mathrm{CO} \rightarrow \mathrm{NH}_{2} \mathrm{CH}_{2} \mathrm{CO}+\mathrm{OH} \\
& \quad \rightarrow \mathrm{NH}_{2} \mathrm{CH}_{2} \mathrm{COOH}(\mathrm{A}) \\
& \quad \mathrm{NH}_{2}+\mathrm{CH} \rightarrow \mathrm{NH}_{2} \mathrm{CH}+\mathrm{CO} \rightarrow \mathrm{NH}_{2} \mathrm{CHCO}+\mathrm{OH} \\
& \quad \rightarrow \mathrm{NH}_{2} \mathrm{CHCOOH} \rightarrow \mathrm{NH}_{2} \mathrm{CH}_{2} \mathrm{COOH}(\mathrm{B}) \\
& \quad \mathrm{CH}_{2}+\mathrm{CO} \rightarrow \mathrm{CH}_{2} \mathrm{CO}+\mathrm{OH} \rightarrow \mathrm{CH}_{2} \mathrm{COOH}+\mathrm{NH}_{2} \\
& \quad \rightarrow \mathrm{NH}_{2} \mathrm{CH}_{2} \mathrm{COOH}(\mathrm{C}) .
\end{aligned}
$$

The most thermodynamically favored of these three mechanisms is mechanism B. Garrod (2013) also proposed four processes that lead to glycine, in which reactions $(\mathrm{E})-(\mathrm{G})$ are the main potential formation routes:

$$
\begin{aligned}
& \mathrm{H}+\mathrm{NHCH}_{2} \mathrm{COOH} \rightarrow \mathrm{NH}_{2} \mathrm{CH}_{2} \mathrm{COOH}(\mathrm{D}) \\
& \mathrm{NH}_{2}+\mathrm{CH}_{2} \mathrm{COOH} \rightarrow \mathrm{NH}_{2} \mathrm{CH}_{2} \mathrm{COOH}(\mathrm{E}) \\
& \mathrm{NH}_{2} \mathrm{CH}_{2}+\mathrm{HOCO} \rightarrow \mathrm{NH}_{2} \mathrm{CH}_{2} \mathrm{COOH}(\mathrm{F}) \\
& \mathrm{NH}_{2} \mathrm{CH}_{2} \mathrm{CO}+\mathrm{OH} \rightarrow \mathrm{NH}_{2} \mathrm{CH}_{2} \mathrm{COOH}(\mathrm{G}) .
\end{aligned}
$$

Another scenario was presented in Bossa et al. (2009, 2010). The authors showed both experimentally and theoretically that a glycine isomer salt, methylammonium methylcarbamate, can be produced in a water-dominated ice from the thermal reaction between carbon dioxide and methylamine $\left(\mathrm{H}_{2} \mathrm{O}: \mathrm{CO}_{2}: \mathrm{CH}_{3} \mathrm{NH}_{2}\right.$ $=10: 3: 0.5$ ) (both compounds have been observed in comets):

$$
\mathrm{CO}_{2}+2 \mathrm{CH}_{3} \mathrm{NH}_{2} \rightarrow\left[\mathrm{CH}_{3} \mathrm{NH}^{+3}\right]\left[\mathrm{CH}_{3} \mathrm{NHCOO}^{-}\right](\mathrm{H})
$$

\section{$\left[\mathrm{CH}_{3} \mathrm{NH}^{+3}\right]\left[\mathrm{CH}_{3} \mathrm{NHCOO}^{-}\right] \rightarrow\left[\mathrm{CH}_{3} \mathrm{NH}^{+3}\right] \quad\left[\mathrm{NH}_{2} \mathrm{CH}_{2} \mathrm{COO}^{-}\right]$} (I).

Then, the isomer salt acts as the glycine salt precursor upon vacuum-ultraviolet (VUV) photolysis. This means that 
experimental observations and theoretical mechanisms can both explain that glycine production in interstellar and cometary ice. Moreover, because it is mainly synthesized within the ice, it seems to be a good working hypothesis that it is embedded in the water-ice matrix.

In order to interpret the observations made by ROSINA, a numerical model was developed to calculate the abundance of glycine in the atmosphere of comet 67P as a function of the distance from the nucleus, and derive its initial abundance in the nucleus. Sublimation of glycine from the nucleus, which is called parent glycine, or from dust particles that are ejected from the nucleus, as well as the release of glycine when water ice sublimates from dust particles, was modeled and compared to observations.

\section{Model}

\subsection{Glycine density profile}

In order to calculate glycine density as a function of distance from the nucleus of 67P, an approach based on Haser's model assumptions (Haser 1957) was used. This model is quite common for studies of the density distribution of compounds in the atmosphere of comets and also in the case of distributed source studies: for instance, $\mathrm{H}_{2} \mathrm{CO}$ in $\mathrm{C} / 1996 \mathrm{~B} 2$ (Hyakutake), (Biver et al. 1999), C/1995 O1 (Hale-Bopp) (Fray et al. 2006) C/2012 F6 (Lemmon) and C/2012 S1 (ISON) (Cordiner et al. 2014), and for HNC in C/2012 S1(ISON) (Cordiner et al. 2014). This implies the following:

- the cometary nucleus has a spherical shape, the emission is homogeneous from the entire nucleus surface;

- the nucleus production is constant over the timescale that is covere by the calculations;

- expansion of molecules from the nucleus is radial and with constant velocity;

- molecules are only destroyed by photochemistry.

This is considered the simplest model for computing the spatial distribution of gaseous molecules in cometary atmospheres, and it is obvious that it does not reflect the complexity of the cometary nucleus and its activity. For instance, it is clear that the nucleus of comet $67 \mathrm{P}$ is not spherical and that the gas production is not homogeneous. However, it is satisfactory as a first approach for an approximate modeling because our goal here is to study relevant production mechanisms rather than computing precise abundances. Haser's model was also used in previous distributed-source studies that were applied to formaldehyde in comet 1P/Halley (Cottin et al. 2004) and C/1995 O1 Hale-Bopp (Fray et al. 2006).

The glycine and the water sublimation rate $\left(\right.$ molec $\left.\mathrm{cm}^{-2} \mathrm{~s}^{-1}\right)$ from the surface can be calculated through the Hertz-Knudsen relation (Miyamoto 1933),

$\frac{1}{A} \frac{\mathrm{d} N_{x}}{\mathrm{~d} t}=\frac{\alpha P_{x} N_{\mathrm{A}}}{\sqrt{2 \pi M_{x} R T_{\mathrm{p}}}}$

$N_{x}$ is a number of molecules, $A$ is the surface from which molecules sublime $\left(\mathrm{cm}^{2}\right), N_{\mathrm{A}}$ is the Avogadro constant $\left(\mathrm{mol}^{-1}\right)$, $R$ is the gas constant $\left(\mathrm{J} \mathrm{mol}^{-1} \mathrm{~K}^{-1}\right)$, and $\alpha$ is the sticking coefficient of the gas molecules to the surface $(0 \leq \alpha \leq 1$, here $\alpha$ is considered to be equal to 1$). M_{x}$ is the molar mass $\left(\mathrm{g} \mathrm{mol}^{-1}\right)$, and $T_{\mathrm{p}}$ is the temperature of a dust particle $(\mathrm{K})$ from which the glycine sublime. Last, $P_{x}$ is the pressure $(\mathrm{Pa})$ calculated from the
Clausius Clapeyron relation,

$P_{x}=P_{\text {ref }} \exp \left(\frac{L}{R}\left(\frac{1}{T_{\text {ref }}}-\frac{1}{T_{\mathrm{p}}}\right)\right)$,

where $P_{\text {ref }}$ and $T_{\text {ref }}$ are the pressure $(\mathrm{Pa})$ and temperature $(\mathrm{K})$ for which the reference measurements were made. $T_{\mathrm{p}}$ is the temperature of the particle $(\mathrm{K})$, and $L$ is the sublimation enthalpy $\left(\mathrm{J} \mathrm{mol}^{-1}\right)$. The values are reported in Table 1 .

By applying Haser's hypotheses and assuming that glycine can be emitted and photolyzed only from the nucleus, a firstdegree differential equation is obtained:

$\frac{\mathrm{d} n_{\mathrm{Gly}}^{\mathrm{Gas}} r^{2}}{\mathrm{~d} r}=\frac{-J_{\mathrm{Gly}}}{v_{\mathrm{gas}}} n_{\mathrm{Gly}} r^{2}$.

Equation (3) corresponds to the simple case of glycine distribution that is emitted only from the nucleus and is then photolyzed. $n_{\text {Gly }}$ is the glycine molecule density profile $\left(\operatorname{molec~} \mathrm{m}^{-3}\right)$, $v_{\text {gas }}$ corresponds to the gas velocity in $\mathrm{m} \mathrm{s}^{-1}, r$ is the distance from the nucleus $(\mathrm{m})$, and $J_{\mathrm{Gly}}$ is the glycine photolysis constant $\left(\mathrm{s}^{-1}\right)$. A production rate of glycine $Q_{\mathrm{Gly}}\left(\operatorname{molec~s}{ }^{-1}\right)$ that is emitted directly from nucleus ices is considered as initial condition.

To simulate a distributed source of glycine in the gas phase that is emitted from the sublimation of solid-state glycine or from the sublimation of glycine that is embedded in water ice on dust particles that are ejected from the nucleus, the following system of equations has to be considered:

$$
\begin{aligned}
\frac{\mathrm{d} n_{\mathrm{Gly}}^{\mathrm{Gr}}}{\mathrm{d} r}= & -\frac{4 \pi}{v_{p}}\left(\frac{3 M_{x}}{4 \pi \rho_{x}}\right)^{2 / 3} \frac{\alpha P_{x} N_{\mathrm{A}}}{\sqrt{2 \pi R T_{\mathrm{p}}}}\left(n_{\mathrm{Gly}}^{\mathrm{Gr}}\right)^{2 / 3}, \\
\frac{\mathrm{d} n_{\mathrm{Gly}}^{\mathrm{Gas}} r^{2}}{\mathrm{~d} r}= & \frac{Q_{\mathrm{Grains}}}{4 \pi v_{p} v_{\mathrm{gas}}} \frac{4 \pi}{v_{p}}\left(\frac{3 M_{x}}{4 \pi \rho_{x}}\right)^{2 / 3} \frac{\alpha P_{x} N_{\mathrm{A}}}{\sqrt{2 \pi M_{x} R T_{\mathrm{p}}}}\left(n_{\mathrm{Gly}}^{\mathrm{Gr}}\right)^{2 / 3} \\
& -\frac{J_{\mathrm{Gly}}}{v_{\mathrm{gas}}} n_{\mathrm{Gly}}^{\mathrm{Gas}} r^{2} .
\end{aligned}
$$

Equation (4) is related to the glycine molecule density profile, $n_{\text {Gly }}^{\mathrm{Gr}}\left(\mathrm{molec}^{-3}\right)$ from dust particles ejected from the nucleus. The Hertz-Knudsen relation $A=4 \pi\left(\frac{3 M_{x}}{4 \pi \rho_{x}}\right)^{2 / 3}$ is injected in this equation, where $M_{x}$ is the glycine or water molecular mass, $\rho_{X}$ is the glycine or water bulk density $\left(\mathrm{g} \mathrm{m}^{-3}\right)$, and $v_{\mathrm{p}}$ is the dust particles velocity $\left(\mathrm{m} \mathrm{s}^{-1}\right)$. Equations (4) and (5) were used to model cases 2 and 3 using the parameters given in Table 1 (glycine) for case 2 and Table 1 (water) for case 3 . In each particle, $\beta$ corresponds to the initial fraction of glycine in dust particles by mass. The calculations were made for equivalent spherical dust particles of pure glycine by calculating an equivalent radius with

$R_{\beta}=\beta\left(\frac{\rho_{\mathrm{p}}}{\rho_{x}}\right)^{1 / 3} R_{0}$,

in which $\rho_{\mathrm{p}}$ represents the particle mass density $\left(\mathrm{g} \mathrm{m}^{-3}\right), \rho_{x}$ is the glycine or water mass density $\left(\mathrm{g} \mathrm{m}^{-3}\right)$, and $R_{0}$ is the initial particle radius. $R_{\beta}$ decreases with distance from the nucleus as glycine or water ice sublimate. For the sublimation of glycine embedded in water ice in dust particles, the equivalent radius is 


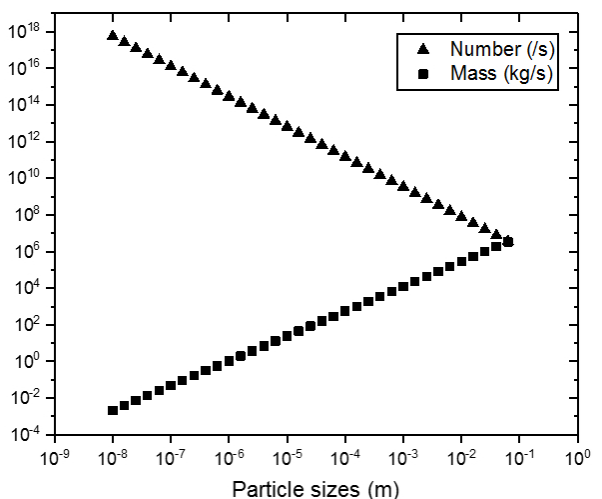

Fig. 2. Particle distribution as a function of particle sizes (m). The triangles represent the number of particles produced per seconds, and the circles show the mass particle production per second $\left(\mathrm{kg} \mathrm{s}^{-1}\right)$ from the cometary nucleus of 67P.

calculated based on a fraction of ice that is present on dust particles instead of the fraction of glycine by mass. To estimate the ice mass $m_{\mathrm{i}}(\mathrm{g})$, a dust-to-ice ratio $d / i$ (estimated to be close to 1 ; Combi et al., in prep.) and the dust mass $m_{\mathrm{d}}(\mathrm{g})$ are considered:

$m_{\mathrm{i}}=\frac{m_{\mathrm{d}}}{d / i}$

Then the fraction of ice is derived by calculating the ratio between the ice mass and the total mass $m_{\mathrm{T}}$ :

$i=\frac{m_{\mathrm{i}}}{m_{\mathrm{T}}}$

The equivalent radius relation is finally obtained using Eq. (6).

Equation (5) is the gaseous glycine density evolution as a function of the distance from the nucleus, $n_{\mathrm{Gly}}^{\mathrm{Gas}}(r)\left(\operatorname{molec~} \mathrm{m}^{-3}\right)$, with a source term that is the glycine sublimation from the dust particles, as well as a sink term that is related to the molecule photolysis. Finally, $v_{\text {gas }}$ corresponds to the gas velocity in $\mathrm{m} \mathrm{s}^{-1}, J_{\mathrm{Gly}}$ is the glycine photolysis constant $\left(\mathrm{s}^{-1}\right)$, and $Q_{\text {Grains }}$ is the number of dust particles that are ejected from the nucleus per second in each size bin. The parameter values are shown in Table 1. It is assumed here that glycine molecules reach the gas velocity immediately after their sublimation, which is a simplification that was also used in Cottin et al. (2004) and Fray et al. (2006). Calculations are made following an Eulerian method.

\subsection{Particle distribution}

In a cometary atmosphere, dust particles do not have the same sizes and masses. The differential equations presented in Sect. 2.1 depend on the temperature and velocity of each particle. Therefore, the distributed sources from a realistic ejected dust particle distribution were computed with their own expansion profiles, that is, with different sizes, temperatures, and velocities.

\subsubsection{Dust production model}

The dust production rate in number for each size range (Fig. 2) was calculated with the power law $\gamma=-1.8 \pm 0.4$ described in Merouane et al. (2017) during the period between August 2014 and the May 2015 equinox. Dust particles from micrometers to centimeters were taken into account. The COmetary Secondary
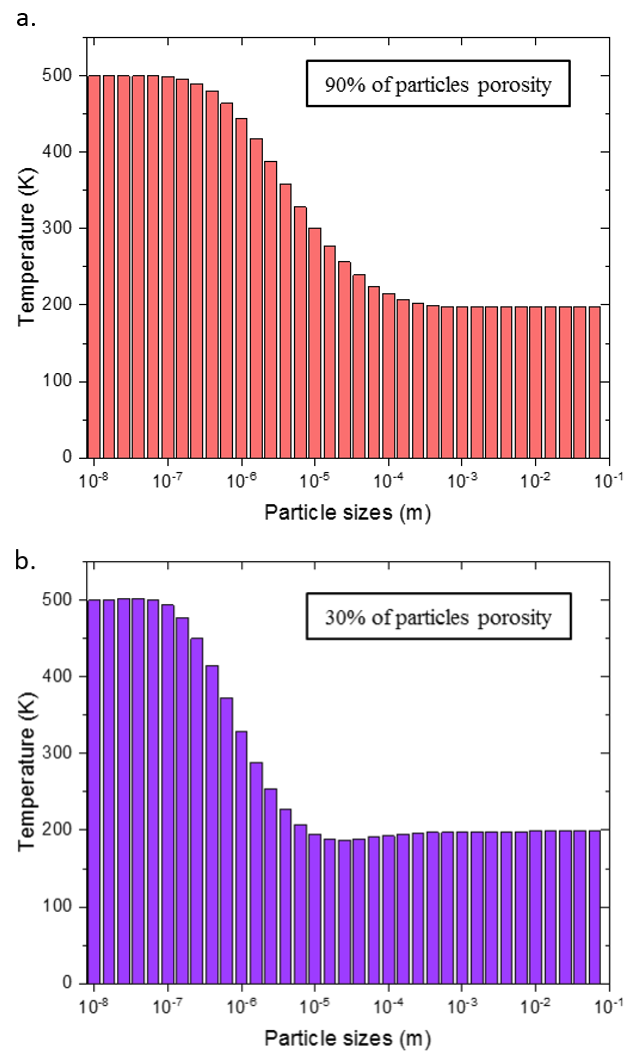

Fig. 3. Calculated profile of particle temperatures as a function of particle sizes at 2 AU from the Sun. Panel a: with $90 \%$ particle porosity. Panel $b$ : with $30 \%$ particle porosity.

Ion MAss spectrometer (COSIMA) has detected dust particles in the micrometer range (Merouane et al. 2016), the Grain Impact Analyser and Dust Accumulator (GIADA) has measured dust particles of a few millimeters (Della Corte et al. 2016; Rinaldi et al. 2017), and the Optical, Spectroscopic and Infrared Remote Imaging System (OSIRIS) has observed dust particles of a few centimeters (Fulle et al. 2010, 2016a).

Figure 2 shows the many small dust particles that correspond to a very low fraction of the total ejected mass. Conversely, there are only a few of the largest dust particles, but they contribute most of the ejected mass.

\subsubsection{Temperatures}

Temperatures (Fig. 3a) were calculated for each class of dust particles with a model based on the Mie scattering theory detailed in Fray et al. (2006). This model takes the size, porosity, and albedo of the dust particles into account. For the albedo, we used the refractive indices of pyroxene $(\mathrm{Mg} 0.6 \mathrm{Fe} 0.4 \mathrm{SiO} 3$; Dorschner et al. 1995) and graphite (Jager et al. 1998). Graphite is representative of black organic matter and provides an upper limit for a strongly absorbing organic component of the grains, and pyroxene represents the silicate component of grains with very low absorption in the visible range.

These temperatures were estimated at $2 \mathrm{AU}$ from the Sun, which is where 67P/Churyumov-Gerasimenko was when the ROSINA glycine measurements were made. Our calculations are also based on dust particles with $70 \%$ in volume of organic matter (Levasseur-Regourd et al. 2018), precisely with amorphous carbon in the model, and with $90 \%$ particle porosity (Hornung et al. 2016). Figure $3 \mathrm{~b}$ was calculated with $30 \%$ porosity. 
Table 2. Initial parameters of the dust model.

\begin{tabular}{|c|c|c|}
\hline Input parameters & Parameter value & Comments \\
\hline \multicolumn{3}{|l|}{ Particle parameters } \\
\hline Particle size (m) & $1 \times 10^{-8}-6.0 \times 10^{-2}$ & \\
\hline Particle density $\left(\mathrm{kg} \mathrm{m}^{-3}\right)$ & 900 & 1 \\
\hline Spheroid aspect ratio, $\mathrm{a} / \mathrm{b}$ & 0.2 & 2 \\
\hline Initial particle orientation (deg) & 45 & 3 \\
\hline \multicolumn{3}{|c|}{ Gas parameters on the initial surface (less than $R_{N} / 50$ above the nucleus surface) } \\
\hline Sound speed $\left(\mathrm{m} \mathrm{s}^{-1}\right)$ & 500 & \\
\hline Gas composition & $10 \times \mathrm{H}_{2} \mathrm{O}+\mathrm{CO}_{2}$ & 4 \\
\hline Gas production rate $\mathrm{Q}_{\text {gas }}\left(\right.$ molec s$\left.^{-1}\right)$ & $2 \times 10^{27}$ & \\
\hline Gas temperature $(\mathrm{K})$ & 100 & \\
\hline Gas density $\left(\mathrm{kg} \mathrm{m}^{-3}\right)$ & $1.0 \times 10^{-6}$ & \\
\hline \multicolumn{3}{|l|}{ 67P/CG parameters } \\
\hline Radius, $R_{N}(m)$ & $2.0 \times 10^{3}$ & (Sierks et al. 2015) \\
\hline Mass, $\mathrm{m}_{\mathrm{N}}(\mathrm{kg})$ & $1.0 \times 10^{13}$ & (Pätzold et al. 2016) \\
\hline Surface temperature, $\mathrm{T}_{\mathrm{N}}(\mathrm{K})$ & 200 & (Capaccioni et al. 2015) \\
\hline
\end{tabular}

Notes. ${ }^{(1)}$ Constrained by the GIADA data (Fulle et al. 2016b, 2017). ${ }^{(2)}$ Compatible with the GIADA measurements (Fulle et al. 2016b, 2017).

${ }^{(3)}$ Compatible with the density used to reproduce particle speeds measured by GIADA (Ivanovski et al. 2017a). ${ }^{(4)}$ All these parameters are derived by the Euler gas flow solutions calibrated with the DCMC 3D+t gas solutions constrained by ROSINA data (Fougere et al. 2016).

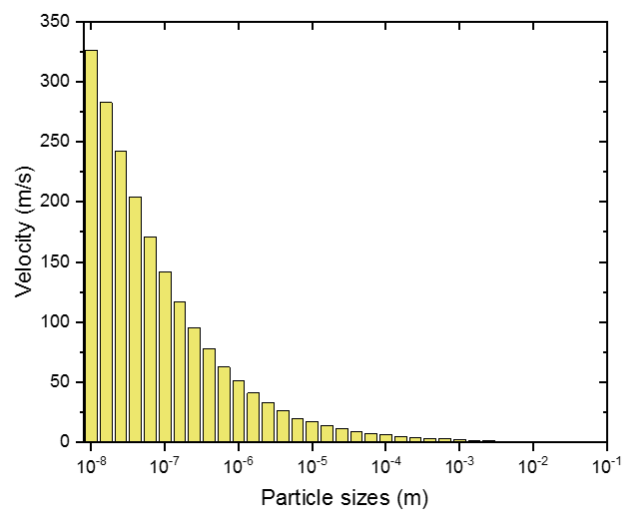

Fig. 4. Particle terminal speeds as a function of particle sizes. The initial properties of the particles are described in Table 2. The particle spheres have a bulk density of $900 \mathrm{~kg} \mathrm{~m}^{-3}$.

\subsubsection{Velocities}

The velocities were calculated using a model for nonspherical dust (Fulle et al. 2015; Ivanovski et al. 2017a,b) by computing the motion of single dust particles in the size range from $10^{-8} \mathrm{~m}$ up to millimeters (Fig. 4). For our simulations we used the physical conditions corresponding to the coma of 67P/CG on 28 March 2015 and GIADA data (Della Corte et al. 2016; Rinaldi et al. 2017). The gas approximation that we used to calculate the aerodynamic force is the Euler approximation for an expanding ideal gas (Anderson \& Wendt 1995, for details), which is analytical. The physical parameters of these gas distributions were adjusted using the gas production rates reported in Fougere et al. (2016). As dust particles, we considered homogeneous isothermal spheres and oblate spheroids with an aspect ratio $\mathrm{a} / \mathrm{b}=0.2$. This elongation value was able to reproduce the GIADA data best (Ivanovski et al. 2017a). Because the measurements on 28 March
2015 were performed close to the nucleus (altitudes from $14 \mathrm{~km}$ up to $26 \mathrm{~km}$ ), the two main forces acting on the dust particles are the aerodynamic force and gravity. In Table 2 we list three sets of parameters: the parameters for $67 \mathrm{P} / \mathrm{CG}$, the assumed physical properties of the simulated dust particles, and the gas flow parameters with the gas production rate. We used a higher gas production rate $\mathrm{Q}_{\text {gas }}\left(2 \times 10^{27}\right.$ molec s$\left.^{-1}\right)$ than was used in the density model (i.e., $5 \times 10^{26}$ molec s$^{-1}$; Biver et al. 2019) with respect to that reported in Table 1 in order to compensate for the nonsphericity of the real expansion. We considered the gas production locally, and in the case of expanding flow, it was derived from numerical calibration using ROSINA data. The maximum liftable dust size under these conditions is a few millimeters (Ivanovski et al. 2017a). The velocity of the large dust particles $\left(10^{-2} \mathrm{~m}\right.$ and larger) is mostly defined by the ejection conditions and remains practically unchanged. In the frame of the spherical expansion model, we cannot estimate their terminal velocity, but based on Fulle et al. (2018), their average velocity is $1.7 \pm$ $0.9 \mathrm{~m} \mathrm{~s}^{-1}$. For instance, in order to have lifted dust particles of $10^{-2} \mathrm{~m}$ and larger, a higher gas production rate should be assumed locally if the total gas production rate still remains the same at $5 \times 10^{26} \mathrm{~s}^{-1}$. For dust sizes $\geq 10^{-2} \mathrm{~m}$ and the physical conditions used in this study, the coefficients Iv and $\mathrm{Fu}$ (Zakharov et al. 2018) show that these dust particles conserve their velocity in the coma. The velocities of oblate spheroids are about twice the velocities of spherical dust particles, which suggests a high sensitivity of the chosen shape to the computed dust velocities.

\section{Results}

Three cases were considered. The first describes glycine that is only emitted as a parent compound from the nucleus and is then photolyzed (Fig. 1a). The two other cases correspond to a distributed source: a first case in which glycine is emitted from the 


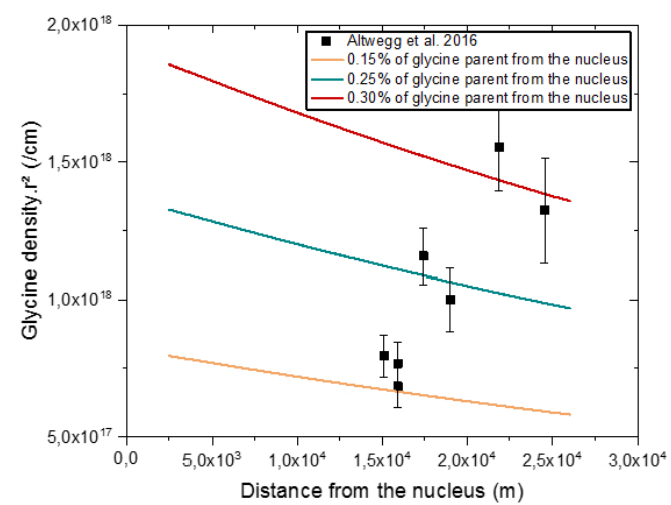

Fig. 5. Glycine density profile as a function of the distance from the nucleus of $67 \mathrm{P}$ when glycine is only emitted from the sublimation of nucleus ice and is then photolysed. The red line corresponds to $0.30 \%$ relative to water of the parent glycine emitted from the nucleus, the blue line corresponds to $0.25 \%$, and the yellow line corresponds to $0.15 \%$.

sublimation of solid-state glycine on the dust particles that are ejected from the nucleus (Fig. 1b), and the other case in which glycine is embedded in water ice and emitted from the sublimation of this water ice from dust particles that are ejected from the nucleus (Fig. 1c).

Figure 5 shows the glycine density profile as a function of distance from the nucleus when glycine does not present a distributed source. Specifically, all figures of this paper show the glycine density multiplied by the square of the distance from the nucleus (converted into centimeters). With this representation, a parent source only from the nucleus and without sinks should be presented as a horizontal trend. The ROSINA measurements clearly show a positive slope while the probe moved away from the nucleus, which is characteristic of a distributed source. Here the hypothetical case of glycine only emitted from the nucleus for different abundances is modeled. A good fit for the first points of the ROSINA measurements corresponds to $0.15 \%$ of glycine relative to water in the nucleus, but the trend does not match the data. A higher glycine percentage, 0.25 and $0.35 \%$, was tested, but it only affects the initial glycine density. The trend is still the same. Regardless of the glycine abundance in the nucleus, it is not possible to fit the ROSINA measurements as a parent compound.

The first case for a distributed source, in which glycine is emitted from the sublimation of solid-state glycine on the dust particles ejected from the nucleus, is shown in Fig. 6 using the parameters as presented in Table 1. Figure 6a shows the evolution of glycine density for different abundances of parent glycine from the nucleus. There are no significant differences within the error bars between no parent glycine and $10^{-3} \%$ parent glycine from the nucleus, while higher percentages of parent glycine, such as $10^{-2} \%$, do not match the ROSINA measurements. Therefore, we consider that the amount of parent glycine is not significant for the distribution we modeled. Consequently, the cases shown in Fig. $6 \mathrm{~b}$ for different abundances of glycine in dust particles were calculated without any parent glycine. To reach an abundance with the same order of magnitude as the measurements, $100 \%$ of glycine in the dust particles is required, which is unrealistic. If the abundance is decreased to $50 \%$, the glycine density is too low compared to the measured density. Moreover, the profile shapes are very different from the measured shape, regardless of the amount of glycine in the dust particles. The kinetics of sublimation of glycine, rather than its amount, is the reason why case 2 seems to be inconsistent with measurements.
Figure 7 shows the result of the modeling in case 3, for which the density profile of glycine was calculated with the same ratio of glycine in the sublimating ices from the nucleus (parent source) and those sublimating from the dust particles (distributed source). This shows that it is possible to match ROSINA data with our model when a distributed source of glycine embedded in water ices on dust particles is considered. A good fit corresponds to an abundance of glycine embedded in water ice on dust particles of about $1.7 \times 10^{-5} \%$ (in mass relative to water). The fit is quite sensitive to parameters such as the porosity of the dust particles, however. As described in Sect. 2.2.1, we considered that the dust particles are made of $70 \%$ in volume of organic matter (Levasseur-Regourd et al. 2018), precisely, amorphous carbon, and with a dust particle porosity of $90 \%$ (Hornung et al. 2016).

The porosity affects the particle temperature. As an example, when the porosity decreases to $30 \%$, dust particles would reach cooler temperatures faster. Results for this case are shown in Fig. 8. The same concentration of glycine in water ice, that is, $1.7 \times 10^{-5} \%$, and also the same percentage of organic matter ( $70 \%$ in volume) was used. Here the glycine density is higher than our first result, meaning that when the dust particles are less porous, the less glycine is required to fit the measurements. Particle velocity also has an influence on glycine density. For example, when all velocities are increased by $20 \%$, about $2.5 \times 10^{-5} \%$ of the glycine that is embedded in water ice would be required to have an equivalently good fit. This means almost $50 \%$ more glycine than for the velocities we used here. We tested the effect of the $\mathrm{H}_{2} \mathrm{O}$ ice mass fraction on the dust particles by changing the dust-to-ice ratio. When it is multiplied by 8 , that is, e. $d / i=8$ to reach some of the higher values published for comet 67P (Fulle et al. 2017), the required glycine density is $4.2 \times 10^{-5} \%$ (420 ppb), which is 2.5 times more than for a d/i equal to 1 . The quality of the fit remains the same and the amount of glycine derived is on the same order of magnitude.

The three computed cases to interpret the atypical distribution of glycine measured by ROSINA are summarized below.

- The first case corresponds to a direct production of glycine from the nucleus does not match the observations.

- The second case takes into account the sublimation of solid-state glycine from dust particles that are ejected from the nucleus and cannot explain the trend of the measurements either. It also requires an unrealistically high quantity of solid-state glycine of a few tens of percent in mass of the ejected dust particles.

- The third case considers that glycine is present within the water ice and is emitted from the sublimation of this ice from dust particles that are ejected from the nucleus. This seems to be a good interpretation. It requires about $170 \mathrm{ppb}$ of glycine on dust particles.

\section{Discussion}

The glycine concentration is about a few hundreds to a few thousands of $\mathrm{ppb}$ in chondrites that present aqueous alteration (Martins \& Sephton 2009). By contrast, in thermal metamorphism chondrites, the glycine concentration is about a few tens to a few hundreds of ppb (Burton et al. 2015). Our modeling tends to an abundance of glycine in $67 \mathrm{P}$ of about $170 \mathrm{ppb}$, which corresponds to the smallest measurement range of glycine detected in aqueous carbonaceous chondrites and to the widest range in chondrites with thermal metamorphism. Studies made by Bardyn et al. (2015) have shown that the materials of $67 \mathrm{P}$ have not undergone aqueous alteration. Furthermore, 


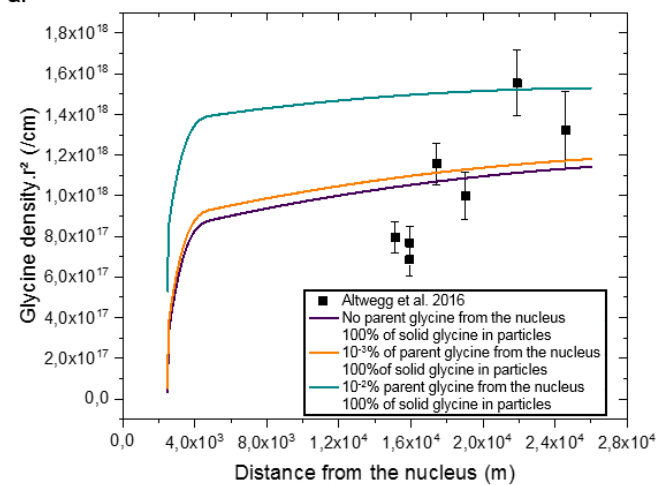

b.

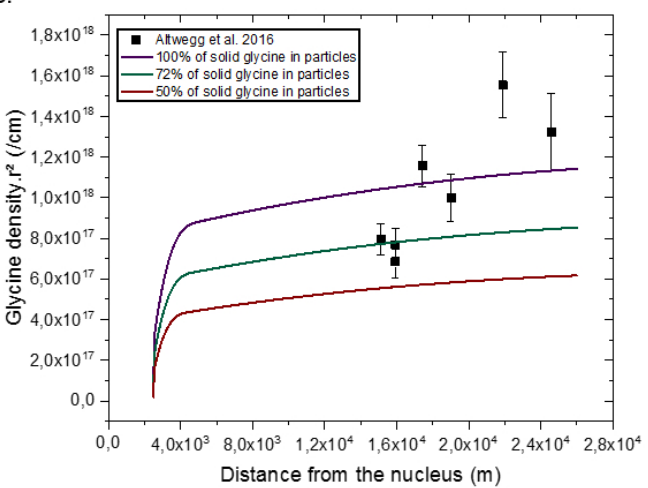

Fig. 6. Density profile of glycine as a function of the distance from the nucleus of $67 \mathrm{P}$ when it presents a distributed source for which glycine is emitted from the sublimation of a solid state on particles. Panel $a$ : with different abundances of parent glycine in the nucleus. Panel $b$ : with different abundances of glycine in particles and no parent glycine from the nucleus.

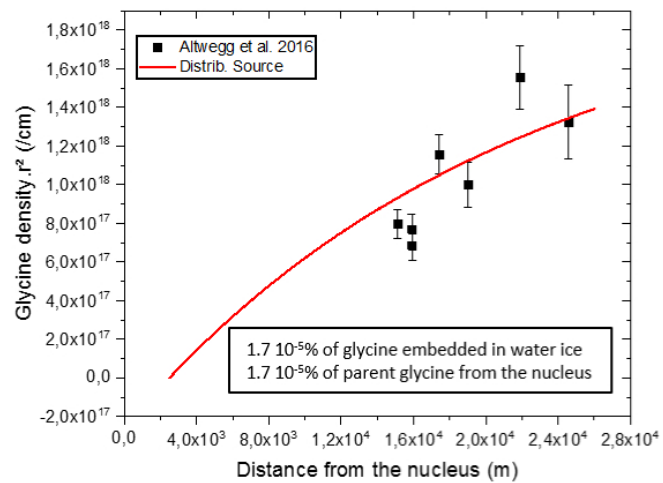

Fig. 7. Density profile of glycine as a function of the distance from the nucleus of 67P when glycine presents a distributed source for which it is embedded in water ice on particles with $1.7 \times 10^{-5} \%$ glycine, and with $1.7 \times 10^{-5} \%$ parent glycine from the nucleus.

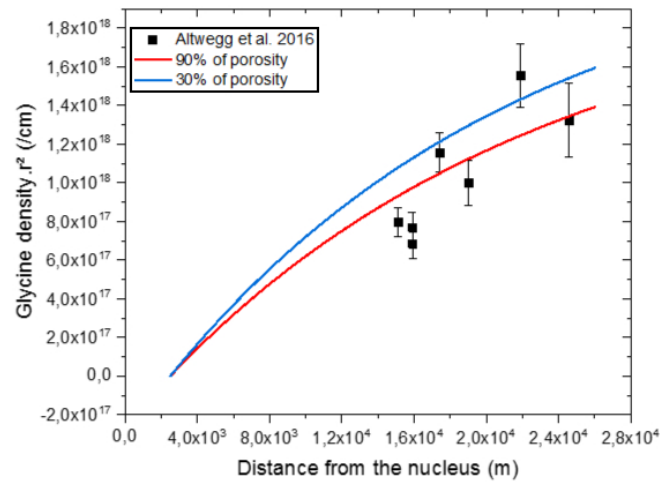

Fig. 8. Density profile of glycine as a function of the distance from the nucleus of $67 \mathrm{P}$ when it presents a distributed source for which glycine molecules are embedded in water ice on particles with $1.7 \times 10^{-5} \%$ glycine and with $1.7 \times 10^{-5} \%$ parent glycine from the nucleus. The blue line corresponds to $30 \%$ porosity, and the red line represents particles with $90 \%$ porosity.

Nuevo et al. (2008) have demonstrated that amino acid quantities are very different for hydrolyzed and non-hydrolyzed fraction residues of photolyzed cometary ice analogs; the concentration is significantly higher in the hydrolyzed fraction. This means that the amount of glycine derived from our modeling is consistent with pristine material that has not been significantly altered either by heat or liquid water.

An important parameter regarding our fits to observations seems to be the temperature. Our modeling is computed at equilibrium temperature, which is not reached immediately at ejection from the nucleus as in our theoretical modeling. The thermal equilibrium time estimated from the heat diffusion inside dust particles (Huebner et al. 2006) can be calculated from

$\tau=\frac{R^{2} \rho c}{\pi^{2} K}$

with $R$ the dust particles radius, $\rho$ their density, $c$ the specific heat, and $K$ the thermal conductivity. Spohn et al. (2015) presented different values measured on the surface by the Philae lander, $\rho=5 \times 10^{-2} \mathrm{~kg} \mathrm{~m}^{-3}, 300 \mathrm{~J}(\mathrm{~kg} \mathrm{~K})^{-1}<c<600 \mathrm{~J}(\mathrm{~kg} \mathrm{~K})^{-1}$, and $0.02 \mathrm{~W}(\mathrm{~m} \mathrm{~K})^{-1}<K<, 0.06 \mathrm{~W}(\mathrm{~m} \mathrm{~K})^{-1}$. As an example, for a particle with a radius of $1 \mathrm{~cm}$, values between 25 and $152 \mathrm{~s}$ are obtained to reach the equilibrium. For the smallest dust particles with millimeter sizes, the equilibrium temperature is reached in about $6 \mathrm{~s}$. For the smallest particles it takes a few fractions of a second.

The dust particles have velocities of between $326 \mathrm{~m} \mathrm{~s}^{-1}$ for the smallest $\left(10^{-8} \mathrm{~m}\right)$ and $1 \mathrm{~m} \mathrm{~s}^{-1}$ for the largest $\left(>10^{-2} \mathrm{~m}\right)$, which means a transport time to reach $14 \mathrm{~km}$ (the first measurement point) of between $42 \mathrm{~s}$ (for the smallest, which reach equilibrium within fractions of second) and $3 \mathrm{~h}$ (for the largest, which reach equilibrium within at most a few minutes). Therefore, the calculated values to reach equilibrium temperature are significantly lower than the transport time from the comet surface to the first ROSINA measurement point. As a first approximation, modeling dust particles at their equilibrium temperature is therefore valid. However, we did not consider the fact that sublimation is an endothermic process that would cool the dust particles as it occurs. Dust particles would therefore be cooler than in our modeling. As shown in Sect. 3, dust particles that reach the coolest temperatures faster would not dramatically influence the amount of glycine that is needed to fit the data.

Because glycine is released at the same time as water ice is sublimating, a distributed source of water and other molecules embedded in water might be detected in the same data set. No such distributed source detection has been reported so far, however. It is interesting to note that De Keyser et al. (2017) have also reported a distributed source for $\mathrm{HCl}$ and $\mathrm{HF}$ due to waterice sublimation from dust particles in the atmosphere of comet 67P. No distributed source of water is detected in their data either, however, because the contribution of sublimating water from the icy dust particles to the total water budget in the coma is suspected to be minor. This is probably also the case for the 28 March 2015 data discussed in this paper: glycine has been measured in a jet of icy dust particles that were ejected from 
the nucleus. We may not have found other distributed source events for glycine because there was only this one fast flyby on 28 March 2015, where the spacecraft changed the radial distance in a short time. For the sublimation of water from dust particles, Gicquel et al. (2016) have suggested based on the brightness profiles of dust jets that icy aggregates can sublimate in the coma of 67P, and that most of this ice sublimates on a length scale of a few tens of kilometers, which is consistent with glycine measurements and our modeling.

This paper is meant to propose a plausible interpretation of the puzzling behavior of glycine in the atmosphere of comet 67P. We suggest a mechanism and an abundance that are quite realistic, but we do not pretend that other mechanisms yet to be conceived might not produce similar matches between observation and theory. For instance, following an approach similar to case 2 , it might be considered that glycine is produced from the degradation of a complex macromolecular organic material. However, to our knowledge, there is no evidence of a macromolecular organic component that would release glycine upon heating. We also consider it unlikely that peptide molecules (polymers of amino acids, in this case, glycine polymers) could be a realistic alternative to the presence of glycine itself. If such peptides compounds were in the dust particles, they would result from the condensation of glycine on itself, and certainly would be order(s) of magnitude less abundant than their precursor (glycine monomer). Data are also lacking for a proper modeling of such a case (both regarding the sublimation of the peptides, depending on their size (dimer, trimers, and polymers), and their kinetics of degradation into glycine). COSIMA, the mass spectrometer that analyzed the solid phase of dust particles (which were captured and analyzed after water ice and volatile are gone), has not reported any detection of molecules such as glycine and other compounds with a rather low volatility (carboxylic acid, aliphatic and aromatic compounds, amines, ketones, etc.) that were expected to be found in the dust particles (Le Roy et al. 2015). The organic matter detected in the dust particles is rather a complex organic network similar to the insoluble organic matter (IOM) observed in carbonaceous chondrites, but in a larger amount (Fray et al. 2016; Bardyn et al. 2017). The hypothesis that compounds such as glycine would be synthesized within the ices and released from the ices as they sublime is consistent with a detection by ROSINA in the gaseous phase and not COSIMA in the solid state. Recent results regarding the detection of aliphatic and aromatic hydrocarbons in comet 67P have shown that many of them are only observed when the coma is dusty (Schuhmann et al. 2019). However, only in March 2015 was a flyby where the spacecraft changed the radial distance in a short time, making this a unique opportunity for distributed sources detection.

Acknowledgements. The authors wish to thank the referee of the paper for their careful reading of the manuscript, questions and comments that contributed to improving the paper. This work has been supported through CNES fundings, in the frame of the Rosetta project. K.H. benefits from a PhD grant from Universite Paris Est Créteil. This work was also supported by the Italian Space Agency (ASI) within the ASI-INAF agreements I/032/05/0 and I/024/12/0. We thank the Rosetta Science Ground Segment at ESAC, the Rosetta Mission Operations Centre at ESOC and the Rosetta Project at ESTEC for their outstanding work enabling the science return of the Rosetta Mission. Rosetta is an ESA mission with contributions from its Member States and NASA.

\section{References}

Altwegg, K., Balsiger, H., Bar-Nun, A., et al. 2016, Sci. Adv., 2, e1600285 Anderson, J. D., \& Wendt, J. 1995, Computational Fluid Dynamics (Berlin: Springer), 206
Bardyn, A., Hilchenbach, M., Briois, C., et al. 2015, AAS/Division Planet. Sci. Meeting Abstracts, 47

Bardyn, A., Baklouti, D., Cottin, H., et al. 2017, MNRAS, 469, S712

Bernstein, M. P., Dworkin, J. P., Sandford, S. A., Cooper, G. W., \& Allamandola, L. J. 2002, Nature, 416, 401

Biver, N., Bockelée-Morvan, D., Crovisier, J., et al. 1999, AJ, 118, 1850

Biver, N., Bockelée-Morvan, D., Hofstadter, M., et al. 2019, A\&A, 630, A19 (Rosetta 2 SI)

Bossa, J.-B., Duvernay, F., Theulé, P., et al. 2009, A\&A, 506, 601

Bossa, J.-B., Borget, F., Duvernay, F., Theulé, P., \& Chiavassa, T. 2010, J. Phys. Org. Chem., 23, 333

Burton, A. S., McLain, H., Glavin, D. P., et al. 2015, Meteorit. Planet. Sci., 50, 470

Capaccioni, F., Coradini, A., Filacchione, G., et al. 2015, Science, 347, aaa0628 Caro, G. M., Meierhenrich, U., Schutte, W., et al. 2002, Nature, 416, 403

Cordiner, M., Remijan, A., Boissier, J., et al. 2014, ApJ, 792, L2

Cottin, H., \& Fray, N. 2008, Space Sci. Rev., 138, 179

Cottin, H., Bénilan, Y., Gazeau, M.-C., \& Raulin, F. 2004, Icarus, 167, 397

Cottin, H., Kotler, J. M., Bartik, K., et al. 2017, Space Sci. Rev., 209, 1

De Keyser, J., Dhooghe, F., Altwegg, K., et al. 2017, MNRAS, 469, S695

De Kruif, C., Voogd, J., \& Offringa, J. 1979, J. Chem. Thermodyn., 11, 651

Della Corte, V., Rotundi, A., Fulle, M., et al. 2016, MNRAS, 462, S210

Dorschner, J., Begemann, B., Henning, T., Jaeger, C., \& Mutschke, H. 1995, A\&A, 300, 503

Elsila, J. E., Glavin, D. P., \& Dworkin, J. P. 2009, Meteorit. Planet. Sci., 44, 1323

Esmaili, S., Bass, A. D., Cloutier, P., Sanche, L., \& Huels, M. A. 2018, J. Chem. Phys., 148, 164702

Fougere, N., Altwegg, K., Berthelier, J.-J., et al. 2016, A\&A, 588, A134

Fray, N., Bénilan, Y., Biver, N., et al. 2006, Icarus, 184, 239

Fray, N., Bardyn, A., Cottin, H., et al. 2016, Nature, 538, 72

Fulle, M., Colangeli, L., Agarwal, J., et al. 2010, A\&A, 522, A63

Fulle, M., Della Corte, V., Rotundi, A., et al. 2015, ApJ, 802, L12

Fulle, M., Marzari, F., Della Corte, V., et al. 2016a, ApJ, 821, 19

Fulle, M., Della Corte, V., Rotundi, A., et al. 2016b, MNRAS, 462, S132

Fulle, M., Della Corte, V., Rotundi, A., et al. 2017, MNRAS, 469, S45

Fulle, M., Blum, J., Green, S., et al. 2018, MNRAS, 482, 3326

Garrod, R. T. 2013, ApJ, 765, 60

Gicquel, A., Vincent, J.-B., Agarwal, J., et al. 2016, MNRAS, 462, S57

Haser, L. 1957, Bull. Soc. Roy. Sci. Liége, 43, 740

Hornung, K., Merouane, S., Hilchenbach, M., et al. 2016, Planet. Space Sci. 133,63

Huebner, W. F., Benkhoff, J., Capria, M.-T., et al. 2006, Heat and Gas Diffusion in Comet Nuclei (Bern: International Space Science Institute)

Ivanovski, S. L., Zakharov, V. V., Della Corte, V., et al. 2017a, Icarus, 282, 333

Ivanovski, S. L., Della Corte, V., Rotundi, A., et al. 2017b, MNRAS, 469, S774

Jager, C., Mutschke, H., \& Henning, T. 1998, A\&A, 332, 291

Kvenvolden, K., Lawless, J., Pering, K., et al. 1970, Nature, 228, 923

Langevin, Y., Hilchenbach, M., Ligier, N., et al. 2016, Icarus, 271, 76

Le Roy, L., Bardyn, A., Briois, C., et al. 2015, Planet. Space Sci., 105, 1

Levasseur-Regourd, A.-C., Agarwal, J., Cottin, H., et al. 2018, Space Sci. Rev., 214, 64

Martins, Z., \& Sephton, M. 2009, Amino Acids, Peptides and Proteins in Organic Chemistry: Origins and Synthesis of Amino Acids (Weinheim: Wiley-VCH Verlag GmbH), 1, 1

Meinert, C., Filippi, J.-J., de Marcellus, P., Le Sergeant d'Hendecourt, L., \& Meierhenrich, U. J. 2012, ChemPlusChem, 77, 186

Merouane, S., Zaprudin, B., Stenzel, O., et al. 2016, A\&A, 596, A87

Merouane, S., Stenzel, O., Hilchenbach, M., et al. 2017, MNRAS, 469, S459

Miyamoto, S. 1933, Trans. Faraday Soc., 29, 794

Nuevo, M., Auger, G., Blanot, D., \& d'Hendecourt, L. 2008, Orig. Life Evol. Biosph., 38, 37

Pätzold, M., Andert, T., Hahn, M., et al. 2016, Nature, 530, 63

Rinaldi, G., Della Corte, V., Fulle, M., et al. 2017, MNRAS, 469, S598

Saiagh, K., Belilla, J., Fray, N., Valorso, R., \& Cottin, H. 2015, Eur. Planet. Sci. Congress, 10

Schuhmann, M., Altwegg, K., Balsiger, H., et al. 2019, A\&A, 630, A31 (Rosetta 2 SI)

Sierks, H., Barbieri, C., Lamy, P. L., et al. 2015, Science, 347, aaa1044

Singh, A., Misra, A., Tandon, P., et al. 2013, Res. Astron. Astrophys., 13, 912

Spohn, T., Knollenberg, J., Ball, A. J., et al. 2015, Science, 349, aab0464

Wagner, W., Riethmann, T., Feistel, R., \& Harvey, A. H. 2011, J. Phys. Chem. Ref. Data, 40, 043103

Woon, D. E. 2002, ApJ, 571, L177

Zakharov, V., Ivanovski, S., Crifo, J.-F., et al. 2018, Icarus, 312, 121 